Research Article

\title{
Evaluation of Changes in Cariogenic Bacteria in a Young Moroccan Population with Fixed Orthodontic Appliances
}

\author{
A. Marda (iD, ${ }^{1}$ S. Elhamzaoui, ${ }^{2}$ A. El Mansari, ${ }^{3}$ K. Souly, ${ }^{4}$ F. Farissi, ${ }^{5}$ M. Zouhdi, ${ }^{4}$ F. Zaoui, ${ }^{6}$ \\ and L. Bahije ${ }^{6}$ \\ ${ }^{1}$ Research Team in Microbiology, Faculty of Medicine and Pharmacy, Mohamed V University, BP 6203, Rabat, Morocco \\ ${ }^{2}$ Department of Microbiology Faculty of Medicine and Pharmacy, Mohamed V University, BP 6203, Rabat, Morocco \\ ${ }^{3}$ Faculty of Dental Medicine Graduate, Mohammed V University, Rabat, Morocco \\ ${ }^{4}$ Laboratory of Microbiology, Ibn Sina University Medical Center of Rabat, Rabat 10100, Morocco \\ ${ }^{5}$ Research Team in Molecular Virology and Oncobiology, Faculty of Medicine and Pharmacy, Mohammed V University, \\ BP 6203, Rabat, Morocco \\ ${ }^{6}$ Department of Research in Biomaterials and Saliva Biomarkers, Department of Dentofacial Orthopedics, \\ Faculty of Dental Medicine, Rabat-Institute, Hospital Center of Ibn-Sina, Mohammed V University (UM5) of Rabat, \\ BP 6212, Madinat Al Irfane, Rabat, Morocco
}

Correspondence should be addressed to A. Marda; asmaa.marda@yahoo.fr

Received 19 July 2018; Revised 5 October 2018; Accepted 4 November 2018; Published 27 November 2018

Guest Editor: Wael Sabbah

Copyright (c) 2018 A. Marda et al. This is an open access article distributed under the Creative Commons Attribution License, which permits unrestricted use, distribution, and reproduction in any medium, provided the original work is properly cited.

Fixed orthodontic appliances hinder the maintenance of proper oral hygiene and result in dental plaque accumulation. Many studies report that qualitative changes in the dental flora occur after initiating the orthodontic treatment, but there is a paucity of literature on the same topic among Moroccan orthodontic patients. The aim of this study was to evaluate the changes of the oral microbial flora during the orthodontic treatment period of a young Moroccan population. Materials and Methods. Dental plaque samples of 18 patients, who were randomly selected before the placement of orthodontic appliances, were collected to isolate and identify the bacterial species involved using classical bacteriological methods for species' culture and identification. The reading was recorded at T0 before placement of the device. New samples were taken again one month later and then three months afterwards, where the readings were recorded as T1 and T2, respectively. The culture was made via Columbia Agar with 5\% sheep blood, Todd Hewitt Broth, and Schaedler medium containing vitamin K3. Bacterial species were identified using API-20 Strep for Streptococci and API-20 A for anaerobic bacteria. The phoenix system was used for identification. Results. After three months of orthodontic treatment, the increase in the frequency of Streptococcus sobrinus and Streptococcus mitis were significant ( 0.01 and 0.02 , respectively) as well as for Lactobacillus (0.03). No significant difference was recorded for other bacterial species. Conclusion. There is a significant qualitative change in oral microorganisms after three months of orthodontic treatment, especially for bacteria that are incriminated in caries formation.

\section{Introduction}

Fixed orthodontic appliances are considered to jeopardize dental health due to the accumulation of oral microorganisms that may cause enamel demineralization, which is clinically visible in the form of white spot lesions [1]. Biofilm formation on fixed orthodontic appliances occurs due to complex interactions between yeast, bacteria, nutrients, and saliva or even serum proteins [2].

Oral Streptococcus is isolated in $50-80 \%$ of orthodontic patients as a common cause of decalcification due to the accumulation of cariogenic plaque around the brackets and its progression into carious lesions in such patients [3]. 
Numerous studies have investigated the influence of orthodontic therapy and appliances on the oral microbial flora. The original aim of the present study was to assess the qualitative changes of cariogenic bacteria in a representative sample of Moroccan young adults.

The placement of fixed orthodontic appliances on teeth results in iatrogenic side effects [2]. During the treatment, dental lesions become difficult to access, resting $\mathrm{pH}$ decreases, the volume of dental plaque is higher, and bacterial flora undergoes qualitative change. All of these factors increase caries risk. Several investigations have shown that changes in the dental flora appear after the initiation of orthodontic treatment [3], such as a higher prevalence of oral streptococci especially Streptococcus mutans and Strepcoccus mitis, which are part of the normal bacterial flora of the oral cavity.

The flora only becomes pathogenic when it is put under circumstances that lead to a frequent and continuous acidification of dental plaque [1] and higher counts of Lactobacillus species, which are closely associated with dental caries. However, there is a scarcity of literature on this topic among Moroccan orthodontic patients.

The aim of the current study was to compare the oral microbial flora among Moroccan patients treated with orthodontic appliances by evaluating the status of this bacterial environment before and after bracket placement.

\section{Materials and Methods}

2.1. Subjects. After a complete oral examination, thorough oral hygiene instructions, and plaque control measures, a total number of eighteen young patients scheduled for orthodontic treatment, age group 16-26, were selected randomly from the Department of Orthodontics, Center of Consultations and Dental Treatments, Rabat, Morocco, during the period from October 2015 to June 2016.

Sampling was carried out via a questionnaire while taking into account the following inclusion criteria: patients with permanent dentition, no clinical signs of periodontitis, no history of systemic diseases, and no administration of antibiotics at least 3 months prior to the treatment. Exclusion criteria included smoking, pregnancy, poor general health, history of periodontal therapy, and antibiotic use.

Dental plaque samples were collected before placement of orthodontic appliances to determine the oral carriage of Streptococci and Lactobacillus in these patients, and the first reading was recorded as T0. After that, the placement of titanium nickel orthodontic wires (G\&H Wire Co., 0.018" $(0.45 \mathrm{~mm}) \mathrm{NiTi}$ wire comprising $55 \%$ nickel and $45 \%$ titanium) and brackets (Ormco Corporation, Glendora, CA, USA) was done by only one operator to avoid interoperator bias. After a period of one month and then three months later, the plaque samples were collected again from the same site, and the second and third readings were recorded as T1 and T2, respectively. The mentioned intervals were chosen based on the assumption that a sufficient bacterial colonization would take place after the placement of orthodontic appliances [1]. All patients had been taught and demonstrated the modified Bass brushing technique using a manual toothbrush (Oral B, Procter \& Gamble, Cincinnati, OH,
USA) and were motivated to meticulously follow throughout the treatment period [4].

2.2. Salivary Microbial Procedures. Dental plaque was collected according to the protocol from the dental surface with a sterile curette followed by rapid transfer of the samples to Amies transport media MAST (DM030) and to anaerobic bacteria culture media (Schaedler broth with vitamin K) [5].

The recipients were immediately sent to the microbiology laboratory where they were centrifuged for 30 seconds and diluted at $1 / 100$ in physiological serum. $50 \mu$ l aliquots were spread on Mitis Salivarius Agar (HiMedia des Laboratoires Pvt. Ltd., Mumbai, India), Columbia agar with 5\% sheep blood, and Schaedler medium containing vitamin $\mathrm{K} 3$ (Schaedler K3 medium, broth, and agar). After 24-48 hours of incubation at $37^{\circ} \mathrm{C}$ for aerobic bacteria and 2-7 days for anaerobic bacteria, the developed colonies were morphologically examined. Each type of colony was subcultured on nonselective growth media to obtain pure cultures. Isolated bacterial colonies were identified based on their morphotinctorial characteristics (Gram-stained smears) and growth characteristics on media, and they also underwent biochemical tests. The species were identified using Rapid ID32 STREP galleries biochemical and enzymatic tests (BioMerieux, SA) for Streptococci and API-20A (BioMerieux, SA) for anaerobic bacteria. To ensure the bacterial identification, we used the automaton Phoenix (Becton Dickinson Phoenix 100 Microbiology Automaton (c Sogemed).

The bacterial strains were preserved in cryotubes by freezing. In each $1.5 \mathrm{ml}$ cryotube, $500 \mu \mathrm{l}$ of bacteria and $500 \mu \mathrm{l}$ of bacterial stock glycerol were mixed together and stored in the freezer $\left(20\right.$ to $\left.80^{\circ} \mathrm{C}\right)$ to use in case further research via molecular diagnostic-genotypic methods was conducted in the future.

2.3. Statistical Analysis. The statistical analysis was performed using the SPSS 13.0 software package. The data were analyzed using descriptive statistics. Fisher's exact test for qualitative variables and the Friedman test for quantitative variables were used to determine the significance of differences between the three groups. The level of statistical significance was set at $P<0.05$.

\section{Result}

3.1. Descriptive Statistics of the Study Population. The study included 18 patients, 11 women $(61 \%)$ and 7 men (39\%). The age was between 16 and 26 years. The average age of all patients was $21.83 \pm 3.48$ years. There was no significant difference between men and women.

3.2. Macroscopic and Microscopic Appearance. The observation of the samples with the naked eye and the optical microscope shows that they are neither hematological nor purulent. After the isolation of the colonies, Gram-positive cocci, Gram-negative bacilli, and Gram-positive bacilli were obtained (Figure 1). 


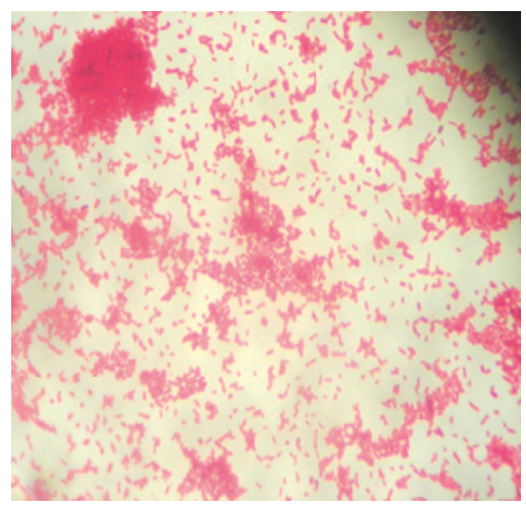

(a)

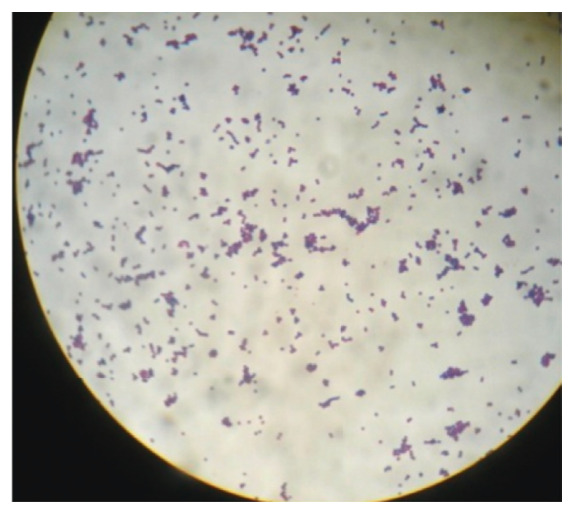

(b)

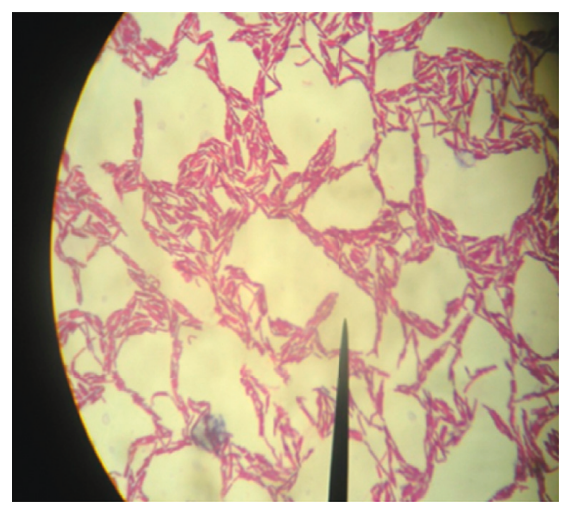

(c)

Figure 1: (a) Gram-negative Bacillus (optical microscope obj. ×100). (b) Gram-positive cocci (optical microscope obj. $\times 100$ ). (c) Grampositive Bacillus (optical microscope obj. $\times 100$ ).

Table 1 presents the frequencies of analyzed bacteria in samples collected from the sample group in T0, T1, and T2.

No significant difference in frequencies was registered for most of the analyzed bacteria, from T0 to T1 and T1 to T2. However, the frequencies of Streptococcus sobrinus and Streptococcus mitis have increased significantly, from T0 to T2 $(P=0.016, P=0.021)$.

As to Lactobacillus, the frequency has increased significantly from T0 to T2 $(P=0.031)$ and insignificantly from $\mathrm{T} 0$ to $\mathrm{T} 1$ and $\mathrm{T} 1$ to $\mathrm{T} 2$. As for enterobacteria, we notice that there is an appearance after 3 months of installation of the fixed orthodontic apparatus of Enterobacter cloacae and Klebsiella pneumoniae (Table 1).

\section{Discussion}

In this study, we found that the frequencies of isolation of bacteria in the oral cavity in patients before orthodontic appliance placement were greater than those measured after 1 month and 3 months of orthodontic appliance placement, respectively. The placement of orthodontic appliances makes it difficult to remove dental plaque by the simple use of a toothbrush and dental floss.

In T0 and T2, we noticed an increase in cariogenic oral microorganisms, especially Streptococcus mitis, Streptococcus sobrinus, and Lactobacillus. This finding was statistically significant $(P=0.021,0.016,0.031)$.

According to Chang et al., the increase in oral Streptococcus following placement of orthodontic appliances can be explained by the irregular nature of their surfaces, which promotes the growth of acidogenic bacteria that prefer to grow on hard surfaces [6]. Batoni et al. showed that orthodontic treatment caused a modification in the oral flora and was associated with elevated counts of cariogenic bacteria in both the dental plaque and saliva [2].

Our finding can be explained by the qualitative change in oral ecology. In other words, it is due to a change in the bacterial composition of dental plaque in patients wearing orthodontic appliances. This result is in line with findings of other studies (Kanaya in 2005 and in $2007[5,6]$ ) which confirmed that orthodontic treatment creates a favorable environment for both quantitative (fast rise in the volume of plaque) and qualitative changes [7]. Kitada et al. in 2009 found in 42 patients, 1 case of Klebsiella pneumoniae and 6 cases of Enterobacter cloacae. In our study, we also distinguished the appearance of these germs after 3 months of fitting the orthodontic apparatus [8]. The literature results show that Klebsiella pneumoniae and Enterobacter cloacae, which are anaerobic and do not need air or dioxygen to function, are among the major causes of halitosis [9]. Rosembloom and Tinanoff found that orthodontic treatment would result in a change in oral flora with a remarkable increase in cariogenic bacteria counts (including oral streptococci) in dental plaque and saliva [10]. In our study, we have seen a proliferation of Streptococcus mitis and Streptococcus sanguinis which are cariogenic bacteria.

Some investigations have presented new data on the duration of microbial salivary changes due to the placement of fixed orthodontic appliances [11]. A significant increase in the cariogenic microorganisms Streptococcus mutans and Lactobacillus in saliva was found after starting fixed orthodontic therapy.

Indeed, these appliances serve as bacterial retention sites. Biofilm formation on the materials used in conservative dentistry and prosthetic dentistry has been investigated by several studies. However, very few studies have examined the interactions between the oral bacteria, particularly Streptococcus mutans, which are the main cariogenic bacteria, and the different materials used in orthodontics.

In vivo studies generally revealed an increase in dental plaque [12] with a specific rise in oral Streptococcus and Lactobacillus counts in plaque and saliva during the orthodontic treatment period [13]. According to these authors, orthodontic appliances are retention sites that promote colonization by certain cariogenic bacteria: oral streptococci and lactobacilli [14]. The characteristics of dental plaque retention on appliances are the cause of a severe development of caries in unusual parts of the teeth, like the vestibular surfaces [15]. 
TABLE 1: Frequency of analyzed bacteria in dental plaque during three phases: before the placement of fixed appliances (T0), one month after the placement of fixed appliances (T1), and three months after the placement of fixed appliances (T2).

\begin{tabular}{|c|c|c|c|c|c|c|}
\hline \multirow{2}{*}{ Bacteria } & T0 & $\mathrm{T} 1$ & $\mathrm{~T} 2$ & \multicolumn{3}{|c|}{$P$-value } \\
\hline & $N(\%)$ & $N(\%)$ & $N(\%)$ & T0 vs T1 & T1 vs T2 & T0 vs T2 \\
\hline Streptococcus sobrinus & $7(38.9)$ & $9(50)$ & $14(77.8)$ & 0.5 & 0.125 & $0.016^{*}$ \\
\hline Streptococcus oralis & $2(11.1)$ & $2(11.1)$ & $3(16.7)$ & 1 & 1 & 1 \\
\hline Streptococcus sanguinis & $2(11.1)$ & $3(16.7)$ & $6(33.3)$ & 1 & 0.375 & 0.219 \\
\hline Streptococcus mitis & $2(11.1)$ & $3(16.7)$ & $10(55.6)$ & 1 & 0.065 & $0.021^{*}$ \\
\hline Streptococcus salivarius & $3(16.7)$ & $3(16.7)$ & $3(16.7)$ & 1 & 1 & 1 \\
\hline Lactobacillus & $2(11.1)$ & $6(33.3)$ & $8(44.4)$ & 1 & 0.63 & $0.031^{*}$ \\
\hline Klebsiella pneumoniae & 0 & 0 & $2(11.1)$ & - & - & - \\
\hline Enterobacter cloacae & 0 & 0 & $3(16.7)$ & - & - & - \\
\hline
\end{tabular}

${ }^{*} P<0.05$ compared to T0.

\section{Conclusion}

Our study consisted of evaluating the quality of bacterial dental plaque before and after the placement of fixed orthodontic appliances on a population of young Moroccan adults. Fixed orthodontic appliances promote the colonization of oral bacteria such as the following cariogenic bacteria: Streptococcus mitis, Streptococcus sobrinus, and Lactobacillus, hence the need to regularly maintain good oral hygiene.

\section{Data Availability}

The data used to support the findings of this study are available from the corresponding author upon request.

\section{Conflicts of Interest}

The authors declare that they have no conflicts of interest.

\section{References}

[1] C. Shukla, R. K. Maurya, V. Singh, and M. Tijare, "Evaluation of changes in Streptococcus mutans colonies in microflora of the Indian population with fixed orthodontics appliances," Dental Research Journal, vol. 13, no. 4, pp. 309-314, 2016.

[2] G. Batoni, M. Pardini, A. Giannotti et al., "Effect of removable orthodontic appliances on oral colonisation by mutans streptococci in children," European Journal of Oral Sciences, vol. 109 , no. 6 , pp. $388-392,2001$.

[3] M. R. Costa, V. C. Silva, M. N. Miqui, T. Sakima, D. M. Spolidorio, and J. A. Cirelli, "Efficacy of ultrasonic, electric and manual toothbrushes in patients with fixed orthodontic appliances," Angle Orthodontist, vol. 77, no. 2, pp. 361-366, 2007.

[4] C. C. Bass, "An effective method of personal oral hygiene," Journal of the Louisiana State Medical Society, vol. 106, no. 2, pp. 57-73, 1954.

[5] T. C. Vizitiu and E. Ionescu, "Microbiological changes in orthodontically treated patients," Therapeutics, Pharmacology and Clinical Toxicology, vol. 4, no. 4, pp. 283-286, 2010.

[6] H. S. Chang, L. J. Walsh, and T. J. Freer, "The effect of orthodontic treatment on salivary flow, $\mathrm{pH}$, buffer capacity, and levels of mutans streptococci and lactobacilli," Australasian Orthodontic Journal, vol. 15, no. 4, pp. 229-234, 1999.

[7] L. Bahije, H. Benyahia, S. El Hamzaoui et al., "Behavior of NiTi in the presence of oral bacteria: corrosion by Streptococcus mutans," International Orthodontics, vol. 9, no. 1, pp. 110-119, 2011.

[8] K. Kitada, A. de Toledo, and T. Oho, "Increase in detectable opportunistic bacteria in the oral cavity of orthodontic patients," International Journal of Dental Hygiene, vol. 7, no. 2, pp. 121-125, 2009.

[9] J. Huang, C.-Y. Li, and J.-H. Jiang, "Effects of fixed orthodontic brackets on oral malodor: a systematic review and meta-analysis according to the preferred reporting items for systematic reviews and meta-analyses guidelines," Medicine, vol. 97, no. 14, article e0233, 2018.

[10] R. G. Rosenbloom and N. Tinanoff, "Salivary Streptococcus mutans levels in patients before, during, and after orthodontic treatment," American Journal of Orthodontics and Dentofacial Orthopedics, vol. 100, no. 1, pp. 35-37, 1991.

[11] K. Peros, S. Mestrovic, S. Anic-Milosevic, and M. Slaj, "Salivary microbial and nonmicrobial parameters in children with fixed orthodontic appliances," Angle Orthodontist, vol. 81, no. 5, pp. 901-906, 2011.

[12] C. Speer, K. Pelz, W. Hopfenmüller, and E.-A. Holtgrave, "Investigations on the influencing of the subgingival microflora in chronic periodontitis. A study in adult patients during fixed appliance therapy," Journal of Orofacial Orthopedics/ Fortschritte der Kieferorthop die, vol. 65, no. 1, pp. 34-47, 2004.

[13] L. Lombardo, Y. Ortan, Ö Gorgun, C. Panza, G. Scuzzo, and G. Siciliani, "Changes in the oral environment after placement of lingual and labial orthodontic appliances," Progress in Orthodontics, vol. 14, no. 1, p. 28, 2013.

[14] T. Vanishree, G. S. Panchmal, R. Shenoy, P. Jodalli, L. Sonde, and N. Kundapur, "Changes in the oral environment after placement of fixed orthodontic appliance for the treatment of malocclusion-a descriptive longitudinal study," Oral Health \& Preventive Dentistry, vol. 15, no. 5, pp. 453-459, 2017.

[15] P. Harikrishnan, T. S. Subha, V. Kavitha, and A. Gnanamani, "Microbial adhesion on orthodontic ligating materials: an in vitro assessment," Advances in Microbiology, vol. 3, no. 1, pp. 108-114, 2013. 


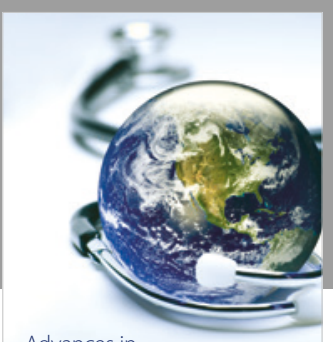

Advances in
Public Health

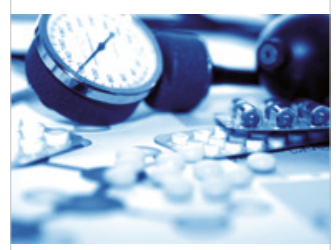

Case Reports in

Medicine

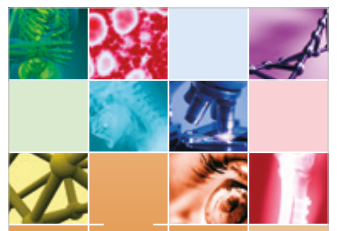

niernational Journal of

Biomaterials
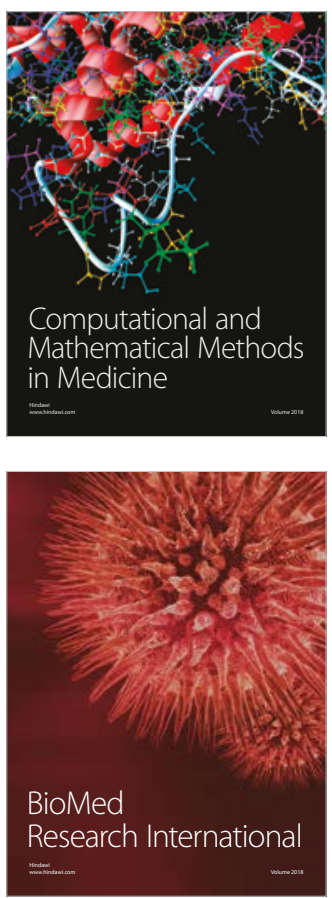

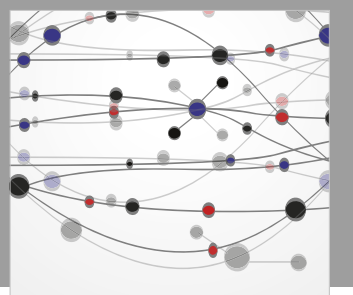

The Scientific World Journal Dentistry

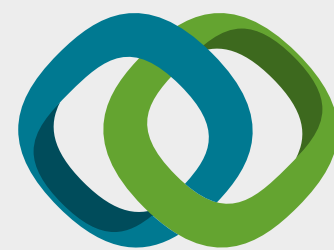

Hindawi

Submit your manuscripts at

www.hindawi.com
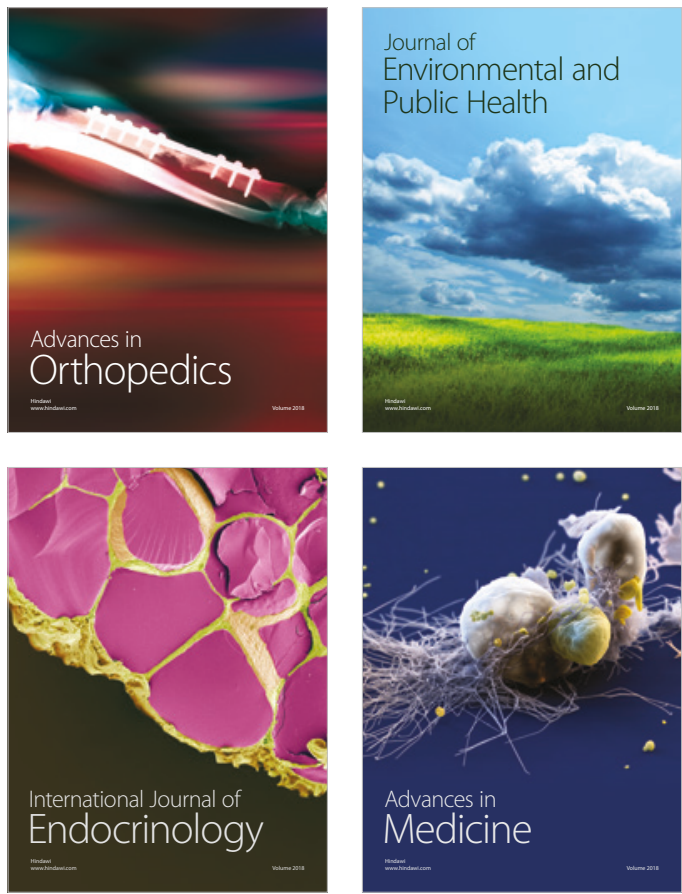
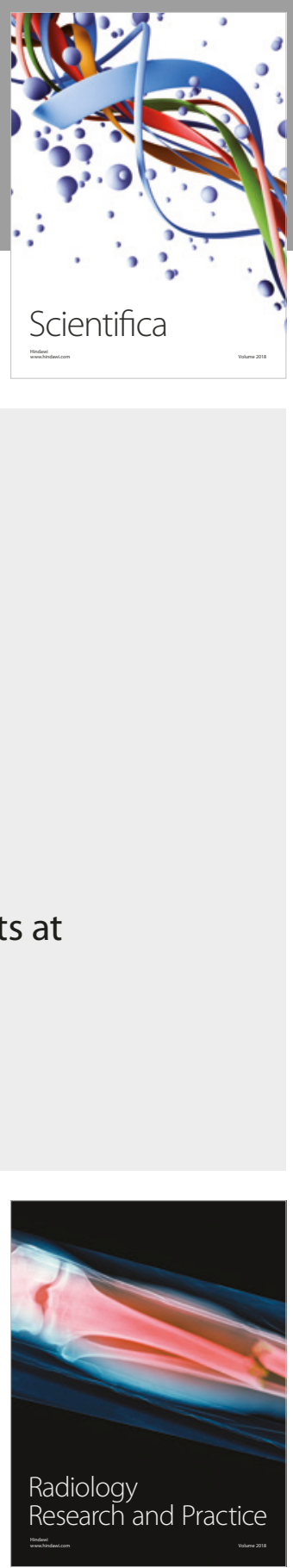

Scientifica

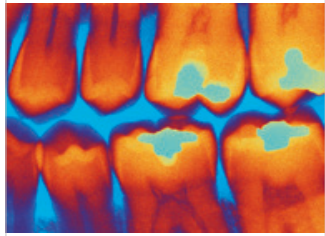

Case Reports in

Dentistry
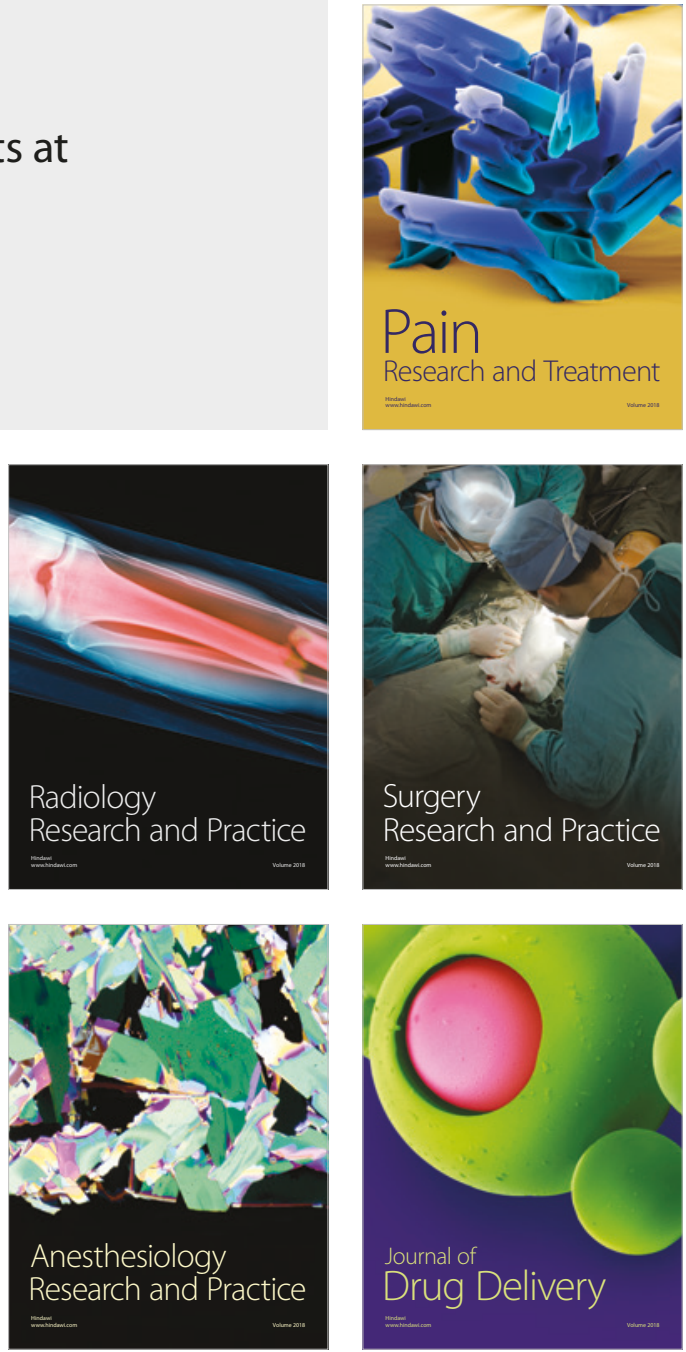